\title{
Fístula Aortoentérica Secundaria A Prótesis De Dacron
}

\author{
${ }^{1}$ Alejandro J Pérez-Alonso, ${ }^{2}$ Carlos del Olmo-Rivas, ${ }^{3}$ Ignacio Machado-Romero, ${ }^{4}$ Patrizio Petrone
}

\section{RESUMEN}

Introducción: La fístula aorto-entérica (FAE) secundaria a prótesis endovascular, es una complicación infrecuente y de extrema gravedad que supone un reto diagnóstico.

Presentación del caso: se trata de un paciente que presentó bruscamente una hemorragia gastrointestinal alta que requirió la práctica urgente de pruebas complementarias que llevaron al diagnóstico de una colección a nivel retroduodenal con burbujas aéreas periprotésicas que apoyaban la existencia de una FAE secundaria.

Conclusión: Aunque el mecanismo exacto de la patogenia es desconocido por el momento, los factores mecánicos e infecciosos parecen ser las causas más frecuentes de esta complicación post-quirúrgica, siendo las teorías expuestas más aceptadas las infecciones bacterianas de la prótesis a nivel de la línea de grapas, daño en el intestino delgado durante la implantación, pseudoaneurismas, y úlceras intestinales.

Palabras clave: Prótesis aórtica, Fístula aorto-entérica, Tratamiento quirúrgico.

How to cite this article: Pérez-Alonso AJ, del Olmo-Rivas C, Machado-Romero I, Petrone P. Fístula Aortoentérica Secundaria A Prótesis De Dacron. Panam J Trauma Crit Care Emerg Surg 2016;5(1):58-60.

Source of support: Nil

Conflict of interest: None

\section{ABSTRACT}

Introduction: The fistula aortoenteric (FAE) secondary to Dacron prosthesis implantation, is a rare and extremely serious complication, that suppose a diagnostic challenge.

Presentation of the case: We report a patient who suddenly presented upper gastrointestinal bleeding that required urgent complementary tests that led to the diagnosis of a retroduodenal periprosthetic collection and air bubbles, supporting the existence of a secondary FAE.

Conclusion: Although the exact mechanism of pathogenesis is unknown for now, mechanical and infectious factors seem

\footnotetext{
${ }^{1-3}$ Attending Surgeon, ${ }^{4}$ Director of Research

1-2Unidad Cirugia HBP. Complejo Hospitalario de Jaén. Universidad de Granada, Granada, Spain

${ }^{3}$ Cirugía, Hospital Quirón Málaga, Málaga, Spain

${ }^{4}$ Department of Surgery, Westchester Medical Center University Hospital, Valhalla, New York, United States
}

Corresponding Author: Patrizio Petrone, Director of Research, Department of Surgery, Westchester Medical Center University Hospital, Valhalla, New York, United States Phone:19144935847, e-mail: patrizio.petrone@gmail.com to be the most common causes of post-surgical complication. The main aacepted theories are: bacterial infections at the staple line level of the prosthesis, damage to the small intestine during implantation, pseudoaneurysms, and intestinal ulcers.

Keywords: Aortic prosthesis, Aortoenteric fistula, Surgical treatment.

\section{INTRODUCCIÓN}

La fístula aorto-entérica (FAE) secundaria a prótesis endovascular, es una complicación infrecuente y de extrema gravedad que supone un desafío diagnóstico, además de una serie de toma de decisiones terapéuticas urgentes. Es importante recordar su existencia en aquellos pacientes que han sido sometidos a cirugía aórtica y que se presenten con fiebre, hemorragia digestiva $u$ otra sintomatología sistémica. ${ }^{1}$

Su curso natural es la defunción por hemorragia y/o sepsis, a menos que un rápido diagnóstico y un tratamiento quirúrgico adecuado eviten el fatal desenlace. Presentamos a continuación un caso de FAE secundaria en un paciente portador de una prótesis aórtica tras reparación aórtica endovascular (endovascular aorta repair, EVAR por sus siglas en inglés). ${ }^{2}$

\section{PRESENTACIÓN DEL CASO}

Se trata de un paciente varón de 72 años de edad, que acudió al Departamento de Urgencias (DU) por presentar un cuadro de malestar general de 20 días de evolución, caracterizado por molestias localizadas en el epigastrio y en la zona periumbilical, asociado con estreñimiento, febrícula y pérdida de peso. Como antecedentes personales presentaba hipertensión arterial de larga evolución, hipercolesterolemia, endarterectomía carotídea bilateral por estenosis superior al 90\% en ambas carótidas internas, y un aneurisma de la aorta abdominal infrarrenal sintomático de 9 años de evolución, el cual fue intervenido mediante EVAR y colocándose una prótesis de Dacron. Se le realizó un análisis sanguíneo en el DU, en el cual se destacaban: hemoglobina 7,4 g/dl (rango: 13,8-17,2), urea $340 \mathrm{gr} / \mathrm{dl}$ (rango: 7-20), y proteína C reactiva, PCR $157 \mathrm{gr} /$ dl (valor normal: hasta 6), por lo se decide su admisión para completar de estudiar al paciente.

Durante su hospitalización, el paciente presentó bruscamente una hemorragia gastrointestinal alta que requirió la práctica urgente de una endoscopia gastroduodenal en la que se observaron restos hemáticos 
desde el estómago hasta la segunda porción duodenal, sin objetivar punto sangrante ni poder avanzar en la exploración. Se realiza una tomografía computarizada (TC) de urgencia con contraste, que mostró cambios inflamatorios en la pared de la aorta abdominal y una colección a nivel retroduodenal con burbujas aéreas periprotésicas que apoyaban la existencia de una FAE secundaria, y sin evidencia de sangrado activo en el momento de la exploración,.

Se inició transfusión sanguínea, seguida de estabilización hemodinámica e intervención quirúrgica urgente. En un primer tiempo se realizó el implante de endoprótesis aorto-monoilíaca con oclusión de ilíaca izquierda, taponando el defecto en la prótesis anterior vía femoral, y bypass fémoro-femoral derechaizquierda. En el mismo tiempo quirúrgico se resecó la fístula aorto-entérica mediante laparotomía subcostal, realizándose movilización completa de marco duodenal, encontrándose el defecto en la tercera porción duodenal, el cual se procedió a reparar mediante una sutura simple y una plastia sero-serosa con yeyuno, más epiploplastia del defecto aórtico (Figuras 1 y 2).

Se tomaron cultivos del área periaórtica y se instauró antibioticoterapia de amplio espectro. El paciente evolucionó favorablemente y fue dado de alta en los días posteriores. Luego de veinte días reingresó en el Servicio de Angiología y Cirugía Vascular por un cuadro febril en relación a la infección del material protésico confirmada mediante TC con contraste y gammagrafía con leucocitos marcados. Se decidió entonces una nueva intervención quirúrgica programada en la que en esa oportunidad se realizó un bypass extra anatómico axilo-femoral a bypass femoro-femoral previo, seguido de la retirada de prótesis y endoprótesis, y ligadura de la aorta a nivel infrarenal.

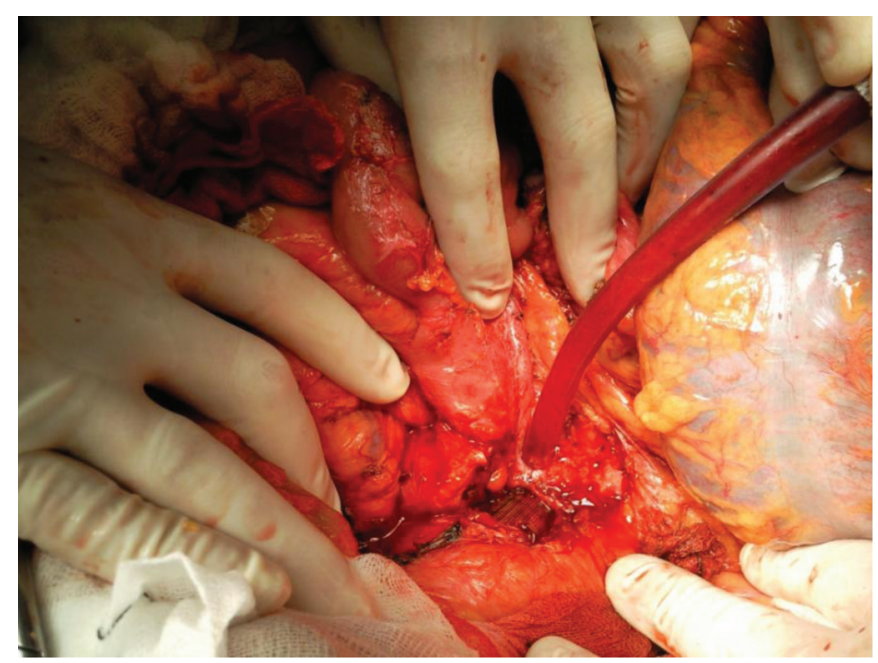

Figura 1: Detalle del defecto en tercera porción duodenal y su comunicación con la luz de la aorta. Puede verse también la prótesis endovascular que estaba implantada previamente

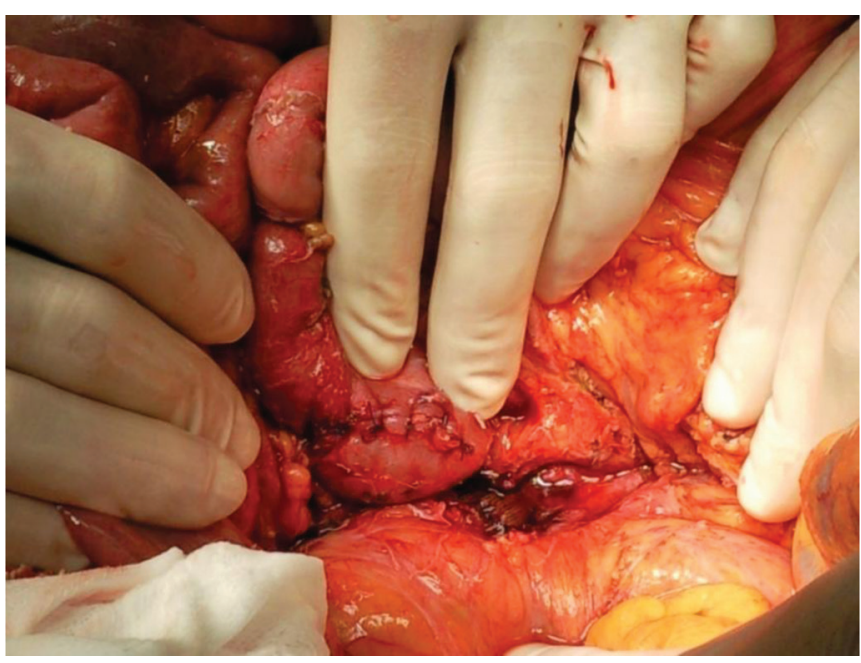

Figura 2: Reparación del defecto intestinal con rafia de puntos intercalados de sutura de monofilamento reabsorbible

Durante el post-operatorio en la Unidad de Cuidados Intensivos presentó un infarto agudo de miocardio anteroseptal, en el contexto de anemia severa y politransfusión, bien tolerado hemodinámicamente salvo episodios aislados de fibrilación auricular paroxística. Evolucionó satisfactoriamente, siendo dado de alta a los quince días de la segunda intervención. En la actualidad el paciente sigue revisiones periódicas a un año tras su intervención.

\section{DISCUSIÓN}

Las FAE secundarias a cirugía aórtica son una complicación rara y potencialmente letal. Su incidencia oscila en el 0,35\% y el 1,6\%. El tiempo medio entre la primera cirugía endovascular y la aparición de las FAE es de 47 meses, pudiendo variar desde los dos días hasta casos reportados a los 24 años. El tiempo medio para su diagnóstico desde el inicio de los síntomas suele ser de ocho días aproximadamente. ${ }^{2,3}$

Aunque el mecanismo exacto de la patogenia es desconocido por el momento, los factores mecánicos e infecciosos parecen ser las causas más frecuentes de esta complicación post-quirúrgica, siendo las teorías expuestas más aceptadas las infecciones bacterianas de la prótesis a nivel de la línea de grapas, daño en el intestino delgado durante la implantación, pseudoaneurismas, y úlceras intestinales. ${ }^{4}$

La hemorragia digestiva alta se ha descrito como el síntoma más frecuente, seguido de los signos de infección como fiebre y síndrome constitucional, y de los síntomas de isquemia aguda por trombosis protésica y artritis sépticas por émbolos, aunque infrecuente. La tríada clásica de dolor abdominal, hemorragia y masa pulsátil se presenta en menos del $25 \%$ de los casos. En cuanto a la localización más frecuentemente afectada 
parece ser la sección entre la tercera y cuarta porciones duodenales. ${ }^{1,5}$

Ninguna técnica diagnóstica puede ser recomendada por sí sola, aunque la combinación de la endoscopia con una técnica de imagen, habitualmente la TC por su amplia disponibilidad, podría ser la mejor opción. En contexto de estudio, la utilización según disponibilidad de arteriografía o leucocitos marcados con $\mathrm{Tc}^{99}$ pueden ser de utilidad. ${ }^{4}$

La existencia de una FAE secundaria implica la muerte del paciente si no se trata con urgencia, e incluso utilizando un tratamiento agresivo los resultados suelen ser pobres. El tratamiento clásico de la FAE consiste en el control de la hemorragia por laparotomía, revascularización extra-anatómica seguido por el cierre de la fístula duodenal y del muñón aórtico infrarrenal, y por último resecándose el injerto infectado. La sustitución in situ de la prótesis aporta aparentemente mejores tasas de mortalidad aunque debe reservarse para situaciones en que la infección esté circunscrita. Los procedimientos intravasculares, menos invasivos no solucionarían el problema de la infección residual, por lo que se emplean como puente a la reparación quirúrgica abierta en pacientes hemodinámicamente inestables con hemorragia activa, permitiendo la estabilización del paciente y la reparación ulterior de la fístula, así como la planificación de la sustitución definitiva de la prótesis mediante un bypass extra-anatómico si persiste la clínica séptica.

También se halla en discusión si ofrece mejores resultados la actuación sobre la fistula entérica tras la colocación de la endoprótesis o actuar en un segundo tiempo, por lo que continúa siendo un tema controvertido con pocas referencias en la literatura y los que existen muestran muy pobres resultados. Los metaanálisis no obtienen resultados significativos por las escuetas series publicadas, el uso de EVAR es reciente y todavía falta experiencia en series a largo plazo. La conclusión más frecuente de los mismos es la necesidad de estudios multicéntricos. ${ }^{5}$

Como corolario, la elección del tratamiento deberá basarse en la situación clínica del paciente, pruebas diagnósticas y experiencia de los equipos quirúrgicos vascular y de cirugía general, acompañado de un equipo multidisciplinar con experiencia en patología vascular.

\section{REFERENCIAS}

1. Simon T, Feller E. Diverse presentation of secondary aortoenteric fistulae. Case Rep Med 2011;2011:406730.

2. Shiraishi M, Kimura C, Yamaguchi A, Adachi H. Secondary aortoenteric fistula: a case report of acute aortic occlusion following cellulitis. Ann Thorac Cardiovasc Surg 2012;18(6): 557-559.

3. Mera-Gallardo O, Gordillo-Escobar E, Luque-Márquez R, Ortiz-Leyva C. Abdominal aortic graft infection secondary to aortoenteric fistula: a diagnostic and therapeutic challenge. Med Intensiva 2013;37(7):493-494.

4. Veraldi GF, Gottin L, Genco B, Bricolo A, Tasselli S, Faggian G, Mazzucco A. Aorto-duodenal fistula on an aortic endograft: a rare cause of late conversion after endovascular aneurysm repair. Gen Thorac Cardiovasc Surg 2012;60(6):350-354.

5. Saratzis N, Saratzis A, Melas N, Ktenidis K, Kiskinis D. Aortoduodenal fistulas after endovascular stent-graft repair of abdominal aortic aneurysms: single-center experience and review of the literature. J Endovasc Ther 2008;15(4):441-448. 